Abstract P085 Table 1 Table demonstrating a comparison of demographic, clinical and prognostic scores between patients assessed via VTA and FTFA. Results from $t$ tests are displayed as mean (SD). Results from Mann Whitney $U$ tests are displayed as median (IQR). Results from Fisher's exact tests are presented as number (\%). Statistical significance was determined by a $p$ value $<0.05$ and signified by *

\begin{tabular}{llllll}
\hline Variable & N & VTA $(n=19)$ & N & FTFA $(n=30)$ & P value \\
\hline Age & 19 & $57.0(49.0-$ & 30 & $61.5(56.8-$ & 0.05 \\
& & $60.0)$ & & $64.3)$ & \\
Sex (male) & 19 & $13(68.4 \%)$ & 30 & $18(60.0 \%)$ & 0.76 \\
Current/Ex-smoker & 19 & $8(42.1 \%)$ & 29 & $14(48.3 \%)$ & 0.77 \\
ARLD & 19 & $9(47.4 \%)$ & 30 & $9(30.0 \%)$ & 0.24 \\
NAFLD & 19 & $2(10.5 \%)$ & 30 & $5(16.7 \%)$ & 0.69 \\
Autoimmune liver diseases (PBC/PSCl & 19 & $6(31.6 \%)$ & 30 & $13(43.3 \%)$ & 0.55 \\
AlH) & & & & & \\
Redo transplantation & 19 & $1(5.3 \%)$ & 30 & $0(0.0 \%)$ & 0.39 \\
HCC & 19 & $0(0.0 \%)$ & 30 & $2(6.7 \%)$ & 0.52 \\
UKELD & 19 & $54.5(5.7)$ & 30 & $54.1(6.0)$ & 0.81 \\
MELD & 19 & $12(10.3-$ & 30 & $10(6.8-$ & 0.10 \\
& & $16.8)$ & & $13.5)$ & \\
CP score & 19 & $8.6(2.7)$ & 30 & $7.8(1.6)$ & 0.24 \\
Time from referral to completion of & 19 & $62(48.0-$ & 28 & $42(21.0-$ & 0.01 * \\
assessment & & $125.0)$ & & $54.5)$ & \\
Patient listed for transplant & 19 & $18(94.7 \%)$ & 30 & $26(86.7 \%)$ & 0.64 \\
\hline & & & & & \\
\hline
\end{tabular}

Initially, face-to-face assessments occurred more frequently as the VTA pathway was established. This reversed with VTA being more common than face-to-face assessments in 2021. There was a trend for patients undergoing VTA to be younger than those undergoing face-to-face assessment. No significant differences were observed when comparing sex, smoking status, disease aetiologies, prognostic scores and listing status. The VTA pathway was associated with a significantly longer time from DIP to full discussion: - this likely reflects that patients were assessed who had been waiting from the prepandemic era via VTA and initial obstacles encountered as part of a new program. No difference was observed in time from listing to transplantation (table 1) - however, long-term outcomes are currently limited.

Conclusion VTA is feasible and will increase access to transplantation. Long-term post-transplant outcome data is required to fully assess the pathway.

\section{P086 THE BILE ACID METABOLISM PATHWAY IS A SIGNIFICANT METABOLIC CHARACTERISTIC OF POSTOPERATIVE RECURRENCE OF HEPATOCELLULAR CARCINOMA UNDERGOING LIVER TRANSPLANTATION BEYOND MILAN CRITERIA}

\footnotetext{
1,2Zijian Zhang ${ }^{*},{ }^{1}$ Yuyuan Chen, ${ }^{1}$ Jian Xu, ${ }^{3}$ Qi Zhou. ${ }^{1}$ Department of General Surgery, The Seventh Affiliated Hospital, Sun Yat-sen University, Shenzhen, China; ${ }^{2}$ Digestive Medicine Center, The Seventh Affiliated Hospital, Sun Yat-sen University, Shenzhen, China; ${ }^{3}$ Department of General Surgery, Huiya Hospital of the First Affiliated Hospital of Sun-Yatsen University, Huizhou, China
}

10.1136/gutjnl-2021-BASL.94
Purpose Liver transplantation is one of the most important treatments for hepatocellular carcinoma (HCC), especially advanced HCC. But the prediction and prevention of postoperative recurrence of hepatocellular carcinoma undergoing liver transplantation beyond Milan criteria are still unknown. More and more evidence showed that metabolic biological progress plays a vital role in initial carcinogenesis and recurrence of HCC. Due to the high risk and lack of donor liver, it is urgent to reveal the mechanism of postoperative recurrence of HCC undergoing liver transplantation beyond Milan criteria. In our research, we used bioinformatics methods to find the metabolic characteristic of postoperative recurrence of hepatocellular carcinoma undergoing liver transplantation beyond Milan criteria.

Method We downloaded the data from Gene Expression Omnibus, TCGA, and normal liver tissues in the GTEx database, and we focused on the biological functions of metabolism-related genes. Firstly, we ran the Limma algorithm to find differential expression genes between recurrence and nonrecurrence cancerous tissue of hepatocellular carcinoma undergoing liver transplantation beyond Milan criteria. Secondly, Gene Set Enrichment Analysis (GSEA) and Gene Set Variant Analysis (GSVA) were utilized to calculate the scores of the differential expression metabolism-related genes and gene sets. Finally, Kaplan-Meier analysis and Cox regression analysis were used to verify the clinical significance of the result. Bioinformatics methods are used to analyze the genomics, transcriptomics, and clinical data.

Results By analyzing the data from GEO, TCGA, and normal liver tissues of GTEx, metabolism-related gene sets were selected for further research. We ran the GSEA algorithm, and we found 'HALLMARK_BILE_ACID_METABOLISM' had the highest enrichment score. And we ran GSVA to confirm the importance of the 'HALLMARK_BILE_ACID_METABOLISM' gene set in HCC. Moreover, it's obvious the higher score of 'HALLMARK BILE ACID METABOLISM' is relevant to the worse overall survival $(\mathrm{P}<0.01)$ and median survival $(\mathrm{P}<0.01)$.

Conclusion Our work revealed the importance of the bile acid metabolism pathway in postoperative recurrence of hepatocellular carcinoma undergoing liver transplantation beyond Milan criteria. It helps us to understand the mechanism of the bile acid metabolism pathway of hepatocellular carcinoma recurrence. It also helps us to predict the recurrence of HCC undergoing liver transplantation beyond Milan criteria. Targeting the bile acid metabolism pathway will be a hopeful strategy to predict and prevent the recurrence of hepatocellular carcinoma after liver transplantation.

\section{P087 LIVER TRANSPLANT IN GLYCOGEN STORAGE DISEASE TYPE 1A; A CASE THAT DISPUTES CURRENT GUIDELINES}

Alexandra Bell*, Ahmed Al-Mukhtar. Sheffield Teaching Hospitals, UK

\subsection{6/gutjnl-2021-BASL.95}

Glycogen Storage Disease Type 1 (GSD1) is a rare autosomal recessive condition with an estimated incidence of around 1 in 100,000 births annually. GSD Type 1a describes 
individuals with defective hepatic glucose-6-phosphatase, an essential enzyme in glucose homeostasis, who suffer with profound fasting hypoglycaemia. Clinical manifestations of the disease include hyperlipidaemia, renal disease and hepatocellular adenomas (HCA). In such patients, hepatic adenoma complications, or severely impaired metabolic control, are uncommon but recognised indications for liver transplantation.

We present the case of a 27-year-old woman with GSD-1a, who suffered with a plethora of secondary complications. Despite a complex dietary regime and extensive adjuvant medical treatment, her metabolic derangements remained resistant to treatment. She was initially referred for liver transplant due to recurrent episodes of life-threatening pancreatitis (secondary to hyperlipidaemia). Ultimately her multiple hepatic adenomas were accepted as indication for transplant. Due to the nature of her indication, her transplant priority was low, and she received an Orthotopic Liver Transplant (OLT) 3 years after referral. Although the adenomas appeared stable on regular pre-transplant MRIs, her explant sample showed histological evidence of hepatocellular carcinoma. Post-transplant, this patient's glucose metabolism and coagulopathy has resolved. She was initially treated for acute cellular rejection with good results. Her renal function remains stable and she reports a much improved quality of life.

Both the European and American College of Medical Genetics (ACMG) GSD1 guidance mentions OLT as a therapeutic option for adenomas suspicious of malignant transformation. This case highlights the shortfalls of using possible HCA transformation as a principle OLT indication. Serum markers are proven to be unreliable in patients with GSD1, and both MRI and CT are poorly predictive of transformation. In waiting for clinical features to develop as suggested by the AMCG, such as rapid enlargement or haemorrhage, patients risk dissemination of disease. The success of OLT in this patient suggests that severely poor metabolic control should be a more prominent recommendation for transplant in GSD1a.

There is evidence detailing post-liver transplant complications in GSD1a, with particular concern over post-operative renal function. Contrasting papers report promising long term outcomes. Due to the rarity of this condition, guidance is based on a handful of small scale case series. Therefore, it is important to document cases such as this one to contribute to GSD1a literature.

\section{REFERENCES}

1. Froissart R, Piraud M, Boudjemline AM, Vianey-Saban C, Petit F, Hubert-Buron A, et al. Glucose-6-phosphatase deficiency. Orphanet J Rare Dis 2011;6:27.

2. Rake JP, Visser G, Labrune P, Leonard JV, Ullrich K, Smit GPA. Guidelines for management of glycogen storage disease type I - European study on glycogen storage. Eur J Pediatr 2002 Oct;161(Suppl 1):S112-9.

3. Kishnani P, Austin S, Abdenur J, et al. Diagnosis and management of glycogen storage disease type I: a practice guideline of the American college of medical genetics and genomics. Genet Med 2014;16:e1.

4. Conti JA, Kemeny N. Type la glycogenosis associated with hepatocellular carcinoma. Cancer 1992;69:1320-1322.

5. Lee P. Glycogen storage disease type I: pathophysiology of liver adenomas. Eur J Pediatr 2002;161:S46-S49.

6. Labrune P. Glycogen storage disease type I: indications for liver and/or kidney transplantation. Eur J Pediatr 2002;161:S53-S55.

\section{P088 DECOMPENSATED CIRRHOSIS CARE BUNDLE QUALITY IMPROVEMENT PROJECT IN LANCASHIRE TEACHING HOSPITALS NHS FOUNDATION TRUST}

${ }^{1}$ Shahzaib Shahzad*, ${ }^{2}$ Amr Elseby, 'Mostafa Afifi. 'Lancashire Teaching Hospital, Chorley, UK; ${ }^{2}$ Lancashire Teaching Hospital, Preston, UK

\subsection{6/gutjnl-2021-BASL.96}

The British Society of Gastroenterology(BSG) in collaboration with the British Association of the Study for Liver disease (BASL) produced a bundle to guide the management of decompensated cirrhotic patients(1) in the first 24 hours, aiming to improve mortality and reduce hospital stays in the United Kingdom. A variation of this guidance was adopted in Lancashire Teaching Hospitals NHS trust(LTHTR).

The aim of this study is to assess the compliance of LTHTR against the decompensated liver cirrhosis care bundle. Retrospective audit performed between December 2019 till March 2020 using data collected from electronic medical records. According to the presentation of decompensation, the management data was plotted against the guidance in the bundle.

The audit identified 35 patients with decompensated cirrhosis. The mean age of patients involved in the audit was $56 \pm 27$, with Male patients $(\mathrm{n}=20)$ more than females $(n=15)$. Some patients had more than one feature of decompensation on admission $(n=15)$. Hospital stays ranged from 3 to 35 days with average of 12.5 days. Ascites was the major presentation of the patients included in this audit $(n=19)$. However, only 5 patients had an ascitic tap done within 8 hours from senior review and 3 had antibiotics. 15 patients had hepatic encephalopathy(severity of which was not mentioned). Majority of those $(\mathrm{n}=11)$ received lactulose. Also, only 8 patients had documentation of Computed tomography (CT) heads considered or dismissed. Hyponatraemia or AKI was the 3rd commonest presentation within the audit's $\operatorname{cohort}(n=11) .9$ patients had a medication review to stop possible drugs leading to AKI. However, only 6 of the patients had resuscitation fluids given on admission. Only 1 patient was weighed on admission, however, 8 patients were monitored for their input and output. Patients who presented with GI bleeds $(n=6)$ had appropriately received platelets and Vit K when needed. Meanwhile, 4 of those patients received prophylactic antibiotics and only 1 received Terlipressin. Sepsis was the least common presentation throughout the audit cohort $(n=4)$. However only 1 had a blood culture done. Out of all the patients that were involved in the audit 12 did not receive Venous Thromboembolism prophylaxis, when it was indicated.

The purpose of such a bundle was to reduce overall hospital stay and all-cause mortality of such patients. The findings were discussed in the local governance meeting which resulted in updating the bundle, establishing teaching sessions for doctors involved in patient management, updating the online system with an easy-to-follow flowchart to manage such patients. A second cycle will follow after implementation 